Renato Musto

\title{
SCIENZA NATURA CAMBIAMENTO
}

\author{
Luigi Maria Sicca \\ a cura di
}

introduzione di

Mario Nicodemi

Editoriale Scientifica

Napoli 
Tutti i diritti sono riservati

(C) 2012 Editoriale Scientifica srl

Via San Biagio dei Librai 39

80138 Napoli

www.editorialescientifica.com

info@editorialescientifica.com

ISBN 978-88-6342-351-8 


\section{Indice}

\section{Prefazione}

SCIENZA, NATURA, CAMBIAMENTO: UNO SGUARDO AL FUTUTRO Luigi Maria Sicca

11 Introduzione

Mario Nicodemi

23 FISICA ROMANTICA

63 Musica e SCIENZA tRA NATURA E CULTURA

83 LA NATURA DEL VIRTUALE

93 CHERNOBYL: LA DURATA DELL'ISTANTE

103 SCIENCE AND DEMOCRACY.

SCIENTIFIC Institutions In AdVANCEd SOCIETIES

123 VECCHIEZZA DEL MONDO E GIOVENTÙ RIBELLE

147 UN RICORDO

Fulvio Musto 